\title{
COVID-19 pneumonia in the emergency department: correlation of initial chest CT findings with short-term outcome
}

\author{
Camila Silva Barbosa ${ }^{1}$ (1) - Guilherme Wilson Otaviano Garcia Chaves ${ }^{1} \cdot$ Camila Vilela de Oliveira $^{1}$. \\ Guilherme Hipolito Bachion ${ }^{1} \cdot$ Chang Kai Chi ${ }^{1} \cdot$ Giovanni Guido Cerri $^{1,2} \cdot$ Thais Carneiro Lima $^{1} \cdot$ Hye Ju Lee ${ }^{1}$
}

Received: 17 July 2020 / Accepted: 9 October 2020 / Published online: 15 October 2020

(C) American Society of Emergency Radiology 2020

\begin{abstract}
Purpose Evaluate chest computed tomography (CT) findings of laboratory-confirmed Coronavirus Disease 2019 (COVID-19) cases and correlate it with clinical and laboratorial signs of severe disease and short-term outcome.

Methods Chest CTs of 61 consecutive cases of COVID-19 disease that attended in our emergency department (ED) were reviewed. Three groups of patients classified according to the short-term follow-up were compared: (1) early-discharged from ED, (2) hospitalized on regular wards, and (3) admitted to intensive care unit (ICU). CT findings were also correlated with clinical and laboratorial features associated with severe disease.

Results Median age was 52 years (IQR 39-63) with male predominance (60.7\%). Most of the patients that did not require hospitalization had parenchymal involvement of less than $25 \%$ on CT $(84.6 \%)$. Among hospitalized patients, interlobular septal thickening and extensive lung disease ( $>50 \%$ of parenchyma) were significantly more frequent in ICU-admitted patients $(P=$ 0.018 and $P=0.043$, respectively). Interlobular septal thickening also correlated with longer ICU stay $(P=0.018)$. Low oxygen saturation $\left(\mathrm{SpO}_{2} \leq 93 \%\right)$ was associated with septal thickening $(P=0.004)$, diffuse distribution $(P=0.016)$, and pleural effusion $(P=0.037)$ on CT. All patients with $>50 \%$ of parenchymal involvement showed $\mathrm{SpO}_{2} \leq 93 \%$. Elevated C-reactive protein (CRP) levels $(>5.0 \mathrm{mg} / \mathrm{dL})$ correlated with consolidation $(P=0.002)$, septal thickening $(P=0.018)$, diffuse distribution $(P=$ $0.020)$, and more extensive parenchymal involvement $(P=0.017)$.

Conclusion Interlobular septal thickening on $\mathrm{CT}$ was associated with ICU admission and longer stay on ICU. Diffuse distribution, septal thickening, and more extensive lung involvement correlated with lower $\mathrm{SpO}_{2}$ and higher CRP levels. Patients that needed hospitalization and ICU admission presented more extensive lung disease on CT.
\end{abstract}

Keywords Coronavirus $\cdot$ COVID-19 $\cdot$ Tomography $\cdot$ Radiology $\cdot$ Patient outcome assessment $\cdot$ Emergency medicine

\section{Introduction}

In December 2019, a Severe Acute Respiratory Syndrome Coronavirus 2 (SARS-CoV-2) outbreak began in Wuhan City, Hubei, China. The disease caused by SARS-CoV-2 was named Coronavirus Disease 2019 (COVID-19). There was a rapid increase in the number of cases and due to the fast geographic spread, and the outbreak was

Camila Silva Barbosa

camila.sbarbosaa@gmail.com

1 Radiology Department, Sírio Libanês Hospital, Sao Paulo, Brazil

2 University of São Paulo (USP), Sao Paulo, Brazil declared a Public Health Emergency of International Concern on January 30, 2020. On March 11, 2020, the World Health Organization named COVID-19 a pandemic. As of August 31, 2020, a total of 25,118,689 cases and 844,312 deaths in 216 countries, areas, or territories have been reported around the world [1].

Coronaviruses are enveloped single-stranded RNA viruses that belong to the order Nidovirales and the family Coronaviridae. Human coronaviruses were first described in 1960, in patients with common cold. Seven species can cause human infections, including $\mathrm{HCoV}$ 229E (229E), HCoV-OC43 (OC43), HCoV-NL63 (NL63), HCoV-HKU1 (HKU1), Severe Acute Respiratory Syndrome Coronavirus (SARS-CoV), Middle East Respiratory Syndrome Coronavirus (MERS-CoV), and the newly described SARS-CoV-2 
(initially named 2019-nCoV). The last three ones belong to the betacoronavirus group, can be highly pathogenic, and are capable to cause severe acute respiratory syndrome and fatal illness [2].

Since it is a novel virus, all humans are susceptible to SARS-Cov-2, with an efficient human-to-human transmission. The incubation period varies from 1 to 14 days (mean 5.2 days) $[3,4]$. The clinical spectrum of COVID-19 is wide and can vary between no symptoms, a common cold, and a severe pneumonia. The main symptoms are fever, dry cough, and dyspnea. Other symptoms include myalgia, headache, chest pain, sore throat, rhinorrhea, diarrhea, nausea, vomiting, and dizziness [5]. A sign of severe disease is hypoxemia.

The diagnosis is based on clinical manifestations, exposure history, and laboratorial tests (real-time RT-PCR-reversetranscriptase polymerase chain reaction or gene sequencing of respiratory or blood specimens). Despite being considered the gold standard, RT-PCR is a test with high specificity (close to 100\%) [6] but low sensitivity (71\%) [7]. Other laboratory findings are reduced white blood cells (especially lymphocytes), increased C-reactive protein (CRP), and erythrocyte sedimentation. According to the Center for Disease Control and Prevention (CDC), greater illness severity has been associated with lymphopenia, neutrophilia, elevated serum alanine aminotransferase and aspartate aminotransferase levels, elevated lactate dehydrogenase, high CRP, high ferritin levels, and high plasma levels of inflammatory markers. Higher mortality rate was associated with elevated D-dimer and lymphopenia. Procalcitonin might also be increased in patients admitted to the ICU $[5,8-10]$.

Imaging in the context of COVID-19 should not be performed as a screening tool for the initial diagnosis. As a novel disease, current recommendations of imaging are conditional and should be part of the diagnostic workup, depending on clinical and laboratory severity and epidemiological data [11]. The imaging modality of choice should be a shared decision involving the referring physician, radiologist, and patient whenever possible. Chest radiography has a lower sensitivity compared with chest CT (0.64 versus 0.92$)$, but it delivers lower radiation doses, is feasible to repeat sequentially, and can be performed with a portable equipment in critical care units. Lung ultrasound involves greater exposure of the healthcare workers, and its diagnostic accuracy has not yet been supported. It might be helpful as a supplemental or alternative modality in specific clinical contexts, involving pregnant women and children for example. Chest CT is the modality with higher sensitivity, as typical features are described to occur in the majority of cases $[12,13]$, although it presents relatively low specificity $(0.56)$ and is especially useful in patients with a pre-existing lung disease. Radiation dose and availability represent limitations for performing CT [11].

Commonly reported chest CT features for COVID-19 pneumonia consisted of peripheral, bilateral, multifocal ground glass opacities (GGO) with or without consolidation and intralobular lines ("crazy-paving"), presence of a reverse halo, or other findings of organizing pneumonia. Non-specific imaging features comprised absence of typical features and presence of non-rounded non-peripheral multifocal, diffuse, perihilar, or unilateral GGO with or without consolidation, lacking a specific distribution. An atypical appearance comprehended isolated lobar or segmental consolidation without GGO, discrete small nodules (centrilobular, "tree-inbud"), lung cavitation, and smooth interlobular septal thickening with pleural effusion [14].

Chest CT has also been performed in the evaluation of disease severity and in the follow-up of patients with COVID-19 $[15,16]$. Radiological evidence of extensive parenchymal disease is described to be related to severe disease, and other CT features suggest a possible correlation with a worse outcome $[15,17]$. This study aimed to review the CT imaging findings of laboratory-confirmed COVID-19 disease cases and correlate the $\mathrm{CT}$ features with short-term outcome and clinical and laboratorial signs of severe illness.

\section{Materials and methods}

\section{Patient selection}

The institutional review board approved this retrospective study, and the requirement of written informed consent was waived. The study included consecutive symptomatic patients assisted at the emergency department (ED) of our institution with COVID-19 disease confirmed by RT-PCR, who underwent non-contrastenhanced chest CT from March 7 to March 30, 2020, inclusive of these days. Patients with insufficient clinical or laboratorial information and those with normal CT findings were excluded. Clinical and laboratorial data, imaging features, and early clinical outcome of sixty-one confirmed cases of COVID-19 were reviewed.

\section{Clinical data collection}

Patients' electronic medical records were reviewed. Demographic information, clinical signs and symptoms, and laboratorial tests (collected in the same day of the CT scan) were recorded.

Based on clinical follow-up information, patients were classified into 3 outcome groups: (1) discharged from ED, not requiring hospitalization (group 1); (2) treated in hospital, but with no need of ICU admission (group 2); and (3) admitted to the ICU at any time during their hospitalization (group 3). The length of stay in hospital was also assessed. 


\section{CT scanning protocol}

Chest CTs were performed using multidetector scanners (Somatom Definition Flash, Somatom Force, and Somatom Definition AS - Siemens Healthcare, Erlangen, Germany). Non-enhanced chest CT scans were acquired with the patient in supine position, arms raised, during end-inspiration breathhold. The acquisition parameters were as follows: tube voltage of $110-140 \mathrm{kV}$, with automatic exposure control, pitch of 0.984 , and slice thickness of $1.0-1.25 \mathrm{~mm}$. Coronal and sagittal images were obtained using the multiplanar reformatting technique on a workstation.

\section{CT image analysis}

Two radiologists ( 2 and 7 years of experience) performed a blinded, independent retrospective review of all CT images. The discrepancies were reviewed by a third radiologist with 12 years' experience in thoracic radiology, which was followed by the establishment of a consensus between the three radiologists.

CT findings were classified according to (1) parenchymal patterns: ground glass opacities (increased attenuation without obscuration of the underlying vessels), consolidation (increased intensity of lung parenchyma, which obscures the underlying vessels), centrilobular micronodules (focal opacity of less than $3 \mathrm{~mm}$ at the bronchovascular core of a secondary pulmonary nodule), bronchial wall thickening, and interlobular septal thickening; (2) distribution: unilateral or bilateral; focal, multifocal or diffuse; (3) location: involvement or spare of each lung lobe; and (4) extension: visual analysis of the degree of lung involvement (less than 25\%, between 25 and $50 \%$ or more than $50 \%$ of parenchymal extension). Presence of pleural effusion and mediastinal lymph node enlargement were also analyzed.

\section{Statistical analysis}

All data was analyzed using SPSS (version 23.0, SPSS Inc., Chicago, IL, USA). Continuous variables are expressed as medians and interquartile range (IQR) and categorical variables as numbers and percentages. The Mann-Whitney $U$ test was used to compare continuous variables, and Fisher's exact test was used to compare categorical variables. Inter-rater reliability (IRR) of CT findings was calculated with Cohen's kappa $(\kappa)$ coefficient, and the result was interpreted as follows: values $\leq 0$ as indicating no agreement, 0.010.20 as none to slight, $0.21-0.40$ as fair, $0.41-0.60$ as moderate, $0.61-0.80$ as substantial, and $0.81-1.00$ as almost perfect agreement. Statistical significance was considered with $P \leq 0.05$.

\section{Results}

\section{Patient demographic and clinical characteristics}

Sixty-one cases with positive RT-PCR for SARS-CoV-2 were analyzed. Median age was 52 years (39-63), with older age among ICU-admitted patients (median 60 years, 54-68) and a male predominance $(60.7 \%)$. The most common symptoms were cough, which occurred in $48 / 61$ patients $(78.7 \%)$, fever in $47 / 61$ (77\%), and dyspnea in 34/61 (55.7\%). The median time since onset of symptoms was 7 days (5-9). Other symptoms are summarized in Table 1.

On vital sign triage, $11 / 61$ patients $(18 \%)$ presented low oxygen saturation $\left(\mathrm{SpO}_{2} \leq 93 \%\right)$ and $3 / 61$ patients $(4.9 \%)$ had an increased respiratory rate ( $R R \geq 24 \mathrm{ipm})$, with only one patient presenting both.

Laboratory blood tests showed leukopenia (leukocyte count $\left.<3500 / \mathrm{mm}^{3}\right)$ in $11 / 61$ patients $(18 \%)$ and lymphopenia (lymphocyte count $<900 / \mathrm{mm}^{3}$ ) in $19 / 61(31.1 \%)$. Elevated CRP levels $(>5.0 \mathrm{mg} / \mathrm{dL})$ were found in $24 / 61$ patients $(39.3 \%)$ (Table 1). Thirty-eight patients also underwent influenza PCR test and had negative results.

\section{Clinical outcome}

Thirteen patients $(21.3 \%)$ did not need hospitalization and were discharged from ED. Among the 48 hospitalized patients, one-quarter required ICU admission. One-third of the patients treated in ICU (4/12) required mechanical ventilation. The median hospitalization time was 10 days (4-12), and median length of ICU stay was 5.5 days (3-16). Two patients stayed in ICU for more than 50 days. None of the patients of this study died.

\section{CT analysis}

GGO occurred in all cases; consolidation was seen in association with GGO in 33/61 cases (54.1\%), and interlobular septal thickening was found in $24 / 61$ cases (39.3\%) (Fig. 1). CT findings were mostly bilateral (91.8\%) and multifocal (90.2\%) (Figs. 1, 2, and 3). Diffuse distribution occurred in only $4 / 61$ cases (6.6\%). Involvement of all pulmonary lobes occurred in about two-thirds of the cases (67.2\%), and among those with fewer lobes involved, the lower lobes were the most frequently affected, with more than $90 \%$ of the total cases presenting opacities in the lower lobes. Most of the patients had little extension of lung disease, with less than $25 \%$ of parenchymal involvement in 35/61 cases $(57.4 \%)$. Only four patients $(6.6 \%)$ had more than $50 \%$ of the lung parenchyma involved (Fig. 3). Mild pleural effusion was seen in five cases $(8.2 \%)$. CT findings are detailed in Table 2. 
Table 1 Demographic characteristics and clinical and laboratorial information

\begin{tabular}{|c|c|c|c|c|c|}
\hline Characteristic & $\begin{array}{l}\text { All patients } \\
(N=61)\end{array}$ & $\begin{array}{l}\text { Group 1 } \\
(N=13)\end{array}$ & $\begin{array}{l}\text { Group 2 } \\
(N=36)\end{array}$ & $\begin{array}{l}\text { Group 3 } \\
(N=12)\end{array}$ & $P$ value* \\
\hline Age (years), median (IQR) & $52(39-63)$ & $50(43-68)$ & $48.5(53.5-69)$ & $60(54-68)$ & 0.018 \\
\hline Age $\geq 65$ years & $13(21.3)$ & $4(30.8)$ & $5(13.9)$ & $4(33.3)$ & 0.199 \\
\hline Male sex & $37(60.7)$ & $8(61.5)$ & $24(66.7)$ & $5(41.7)$ & 0.176 \\
\hline \multicolumn{6}{|l|}{ Symptoms } \\
\hline Days from symptoms onset, median (IQR) & $7(5-9)$ & $7(5-9)$ & $7(3.5-9)$ & $7(5-9.5)$ & 0.756 \\
\hline Cough & $48(78.7)$ & $11(84.6)$ & $30(83.3)$ & $7(58.3)$ & 0.113 \\
\hline Fever & $47(77)$ & $7(53.8)$ & $30(83.3)$ & $10(83.3)$ & 1.000 \\
\hline Dyspnea & $34(55.7)$ & $9(69.2)$ & $20(55.6)$ & $5(41.7)$ & 0.511 \\
\hline Myalgia & $22(36.1)$ & $4(30.8)$ & $11(30.6)$ & $7(58.3)$ & 0.101 \\
\hline GI Symptoms (nausea, vomiting, diarrhea) & $18(29.5)$ & $2(15.4)$ & $11(30.6)$ & $5(41.7)$ & 0.500 \\
\hline Coryza & $17(27.9)$ & $7(53.8)$ & $9(25.0)$ & $1(8.3)$ & 0.414 \\
\hline Sore throat & $8(13.1)$ & $1(7.7)$ & $4(11.1)$ & $3(25.0)$ & 0.345 \\
\hline Chest pain & $7(11.5)$ & $1(7.7)$ & $5(13.90$ & $1(8.3)$ & 1.000 \\
\hline \multicolumn{6}{|l|}{ Clinical signs } \\
\hline Crackling & $24(39.3)$ & $2(15.4)$ & $15(41.7)$ & $7(58.3)$ & 0.341 \\
\hline Wheezing & $2(3.3)$ & $1(7.7)$ & $1(2.8)$ & $0(0.0)$ & 1.000 \\
\hline \multicolumn{6}{|l|}{ Vital signs } \\
\hline RR (ipm), median (IQR) & $17(16-19)$ & $17(16-18)$ & $17(16-18)$ & $18(16-21.5)$ & 0.155 \\
\hline $\mathrm{RR} \geq 24 \mathrm{ipm}$ & $3(4.9)$ & $0(0)$ & $1(2.8)$ & $2(16.7)$ & 0.150 \\
\hline $\mathrm{SpO}_{2}(\%)$, median (IQR) & $95(94-97)$ & $98(97-98)$ & $95(94-96)$ & $93.5(91-95.5)$ & 0.026 \\
\hline $\mathrm{SpO}_{2} \leq 93 \%$ & $11(18.0)$ & $0(0)$ & $5(13.9)$ & $6(50)$ & 0.018 \\
\hline \multicolumn{6}{|l|}{ Laboratory findings } \\
\hline CRP (mg/dL), median (IQR) & $3.32(1.03-8.66)$ & $1.29(1.00-4.84)$ & $2.75(0.94-6.85)$ & $10.35(5.81-13.33)$ & 0.001 \\
\hline $\mathrm{CRP}>5.0 \mathrm{mg} / \mathrm{dL}$ & $24(39.3)$ & $3(23.1)$ & $12(33.3)$ & $9(75)$ & 0.018 \\
\hline Lymphopenia $\left(<900 / \mathrm{mm}^{3}\right)$ & $19(31.1)$ & $2(15.4)$ & $9(25)$ & $8(66.7)$ & 0.015 \\
\hline Lymphocytosis $\left(>2900 / \mathrm{mm}^{3}\right)$ & $1(1.6)$ & $1(7.7)$ & $0(0)$ & $0(0)$ & - \\
\hline Leukopenia $\left(>3500 / \mathrm{mm}^{3}\right.$ ) & $11(18)$ & $1(7.7)$ & $9(25)$ & $1(8.3)$ & 0.414 \\
\hline Leukocytosis $\left(>10,500 / \mathrm{mm}^{3}\right)$ & $3(4.9)$ & $1(7.7)$ & $1(2.8)$ & $1(8.3)$ & 0.441 \\
\hline \multicolumn{6}{|l|}{ Clinical outcome } \\
\hline Total hospitalization days, median (IQR) & $10(4-12)$ & $0(0)$ & $7(4-11)$ & $14.5(12-28.5)$ & 0.000 \\
\hline Days in the ICU, median (IQR) & $5.5(3.0-16.0)$ & $0(0)$ & $0(0)$ & $5.5(3.0-16.0)$ & - \\
\hline Mechanical ventilation & $4(6.6)$ & $0(0)$ & $0(0)$ & $4(33.3)$ & - \\
\hline Death & $0(0)$ & $0(0)$ & $0(0)$ & $0(0)$ & - \\
\hline
\end{tabular}

Except where noted otherwise, data are number (\%) of patients. Group 1: discharged patients; group 2: patients hospitalized in general wards; group 3: patients admitted to ICU

$G I$ gastrointestinal, $R R$ respiratory rate, $\mathrm{SpO}_{2}$ peripheral capillary oxygen saturation, $C R P$ C-reactive protein, $I C U$ intensive care unit

$* P$ value refers to group 2 vs group 3

Italicized numbers represent statistically significant $P$ values

The inter-rater reliability was almost perfect for evaluation of disease extension $(k=0.88)$ and at least substantial for all parenchymal patterns ( $k=0.80$ to 1.00 ). The analysis of disease distribution (focal, multifocal, or diffuse) also demonstrated substantial agreement between the radiologists $(k=0.71)$.

Most of the discharged patients (group 1) had less than $25 \%$ of pulmonary involvement on CT (11/13, $84.6 \%)$, which occurred in half $(24 / 48)$ of the hospitalized patients (groups 2 and 3) $(P=0.030)$.
Pulmonary opacities with extension greater than $50 \%$ and the presence of interlobular septal thickening were more frequently seen in ICU-admitted patients (group 3 ) than in patients hospitalized on general wards (group 2) $(P=0.043$ and $P=0.018$, respectively) (Fig. 3). The length of ICU stay was also longer (11 vs 2 days, $P=0.018$ ) in patients with interlobular septal thickening on CT.

CT findings associated with low $\mathrm{SpO}_{2}(\leq 93 \%)$ were interlobular septal thickening $(P=0.004)$, diffuse distribution $(P=$ 


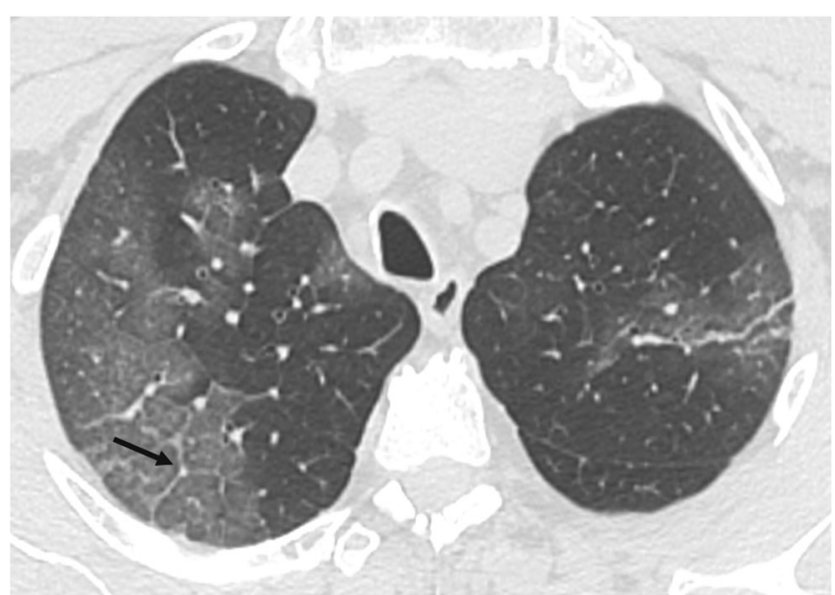

Fig. 1 Chest CT axial image of a 58-year-old man. Bilateral GGO with peripheral distribution and interlobular septal thickening (arrow)

0.016), pleural effusion ( $P=0.037)$, and greater disease extension $(P=0.042$ for $>25 \%$ of parenchyma involvement). High CRP levels $(>5 \mathrm{mg} / \mathrm{dL})$ were associated with consolidation $(P=0.002)$, interlobular septal thickening $(P=0.018)$, diffuse distribution $(P=0.020)$, and parenchymal involvement greater than $25 \%(P=0.017)$. Lymphopenia did not correlate with the analyzed CT features of this study (Table 3 ).

\section{Discussion}

ICU admission rate achieved up to one-quarter of hospitalized patients in previous reports [8,18-23], and despite the differences in the populations and possible variations on clinical practices and admission criteria, we found similar results in our study. Early recognition of severe cases that might need advanced respiratory support in the ICU could improve the management and reduce the mortality rate of patients with COVID-19.

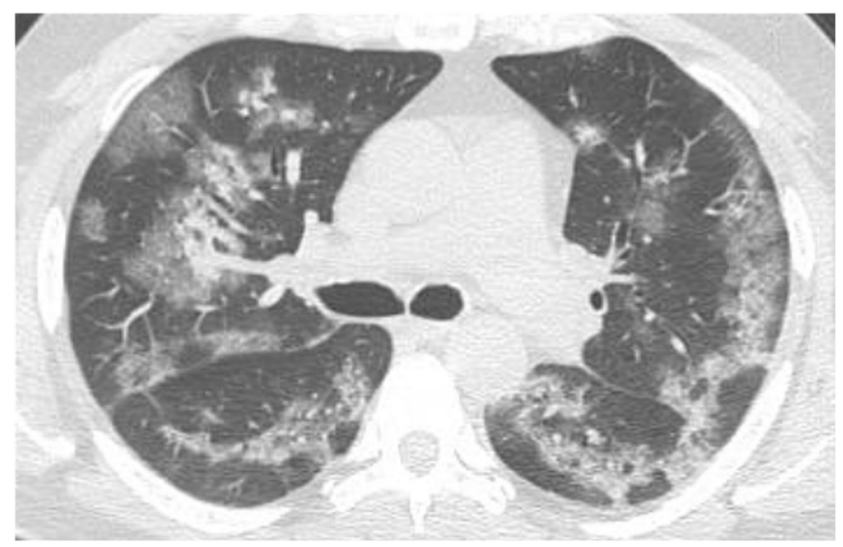

Fig. 2 Chest CT axial image of a 55-year-old man. Bilateral and multifocal rounded GGO, with involvement of $25-50 \%$ of the lung parenchyma

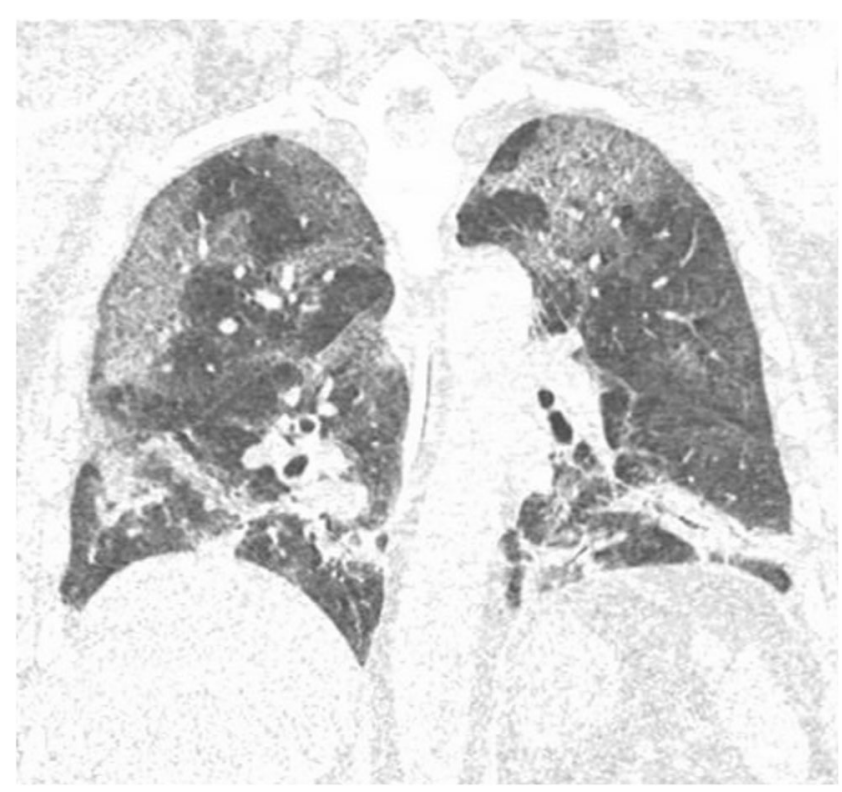

Fig. 3 Chest CT image of a 63-year-old man in coronal view. Extensive GGOs and consolidations with diffuse distribution and involvement of more than $50 \%$ of lung parenchyma

In the largest cohort of COVID-19 patients from China, with 44,672 cases, increased respiratory rate, hypoxemia, and/or $>50 \%$ lung infiltrate extension were defined as features of severe illness [20]. CRP values are significantly higher in patients with severe COVID-19 and have been studied as an early predictor of the disease course [10, 24-26]. Lymphopenia is believed to represent a defective immune response to the virus, and a recent meta-analysis reported its presence in $35-75 \%$ of patients. In our study, it occurred in $31.1 \%$ of the cases. The reason for this variability is probably multifactorial, involving virus genomic mutation as the pandemic expands into different countries, geographic differences, and the time of testing since the beginning of symptoms $[27,28]$. Lymphopenia and leukopenia are more prominent in patients with severe than those with non-severe disease $[8,10$, 26, 29]. In our study, patients referred to ICU presented with higher rates of low $\mathrm{SpO}_{2}(50 \%$ vs $13.9 \%, P=0.018)$, lymphopenia $(66.7 \%$ vs $25 \%, P=0.015)$, and elevated CRP $(P=$ 0.001 ) when compared with ward patients.

More extensive parenchymal impairment, probably representing more alveolar and interstitial pulmonary injury, has been associated with severe disease $[15,17,30]$. In our study, the extension of lung involvement was based on a visual analysis, and, despite not being a quantitative method, we found an almost perfect agreement between readers $(\kappa=0.88)$. More extensive disease correlated with the need of hospitalization and ICU admission; involvement of less than $25 \%$ of lung parenchyma was significantly more frequent in discharged patients, and parenchymal involvement greater than $50 \%$ correlated with ICU admission. Among other analyzed CT findings, 
Table 2 CT characteristics according to outcome

\begin{tabular}{|c|c|c|c|c|c|c|c|}
\hline Characteristic & $\begin{array}{l}\text { All patients } \\
(N=61)\end{array}$ & $\begin{array}{l}\text { Group 1 } \\
(N=13)\end{array}$ & $\begin{array}{l}\text { Hospitalized } \\
\text { patients* } \\
(N=48)\end{array}$ & $P$ value $^{\mathrm{a}}$ & $\begin{array}{l}\text { Group } 2 \\
(N=36)\end{array}$ & $\begin{array}{l}\text { Group } 3 \\
(N=12)\end{array}$ & $P$ value ${ }^{\mathrm{b}}$ \\
\hline \multicolumn{8}{|l|}{ Parenchymal features } \\
\hline GGO & $61(100)$ & $13(100)$ & $48(100)$ & - & $36(100)$ & $12(100)$ & - \\
\hline Consolidation & $33(54.1)$ & $6(46.2)$ & $27(56.3)$ & 0.547 & $19(52.8)$ & $8(66.7)$ & 0.510 \\
\hline Septal thickening & $24(39.3)$ & $3(23.1)$ & $21(43.8)$ & 0.215 & $12(33.3)$ & $9(75.0)$ & 0.018 \\
\hline Bronchial wall thickening & $18(29.5)$ & $3(23.1)$ & $15(31.3)$ & 0.737 & $13(36.1)$ & $2(16.7)$ & 0.292 \\
\hline Centrilobular micronodules & $0(0)$ & $0(0)$ & $0(0)$ & - & $0(0)$ & $0(0)$ & - \\
\hline \multicolumn{8}{|l|}{ Distribution } \\
\hline Unilateral & $5(8.2)$ & $2(15.4)$ & $3(6.3)$ & - & $3(8.3)$ & $0(0)$ & - \\
\hline Bilateral & $56(91.8)$ & $11(84.6)$ & $45(93.8)$ & 0.287 & $33(91.7)$ & $12(100)$ & 0.563 \\
\hline Focal & $2(3.3)$ & $1(7.7)$ & $1(2.1)$ & 0.384 & $1(2.8)$ & $0(0)$ & 1.000 \\
\hline Multifocal & $55(90.2)$ & $12(92.3)$ & $43(89.6)$ & 1.000 & $33(91.7)$ & $10(83.3)$ & 0.587 \\
\hline Diffuse & $4(6.6)$ & $0(0)$ & $4(8.3)$ & 0.569 & $2(5.6)$ & $2(16.7)$ & 0.257 \\
\hline \multicolumn{8}{|l|}{ Location } \\
\hline Right upper lobe & $46(75.4)$ & $8(61.5)$ & $38(79.2)$ & 0.275 & $28(77.8)$ & $10(83.3)$ & 1.000 \\
\hline Right medium lobe & $45(73.8)$ & $9(69.2)$ & $36(75.0)$ & 0.728 & $24(66.7)$ & $12(100)$ & 0.023 \\
\hline Right lower lobe & $57(93.4)$ & $12(92.3)$ & $45(93.8)$ & 1.000 & $33(91.7)$ & $12(100)$ & 0.563 \\
\hline Left upper lobe & $54(88.5)$ & $11(84.6)$ & $43(89.6)$ & 0.634 & $32(88.9)$ & $11(91.7)$ & 1.000 \\
\hline Left lower lobe & $58(95.1)$ & $13(100)$ & $45(93.8)$ & 1.000 & $33(91.7)$ & $12(100)$ & 0.563 \\
\hline All lobes & $41(67.2)$ & $8(61.5)$ & $33(68.8)$ & 0.741 & $23(63.9)$ & $10(83.3)$ & 0.292 \\
\hline \multicolumn{8}{|l|}{ Extension } \\
\hline$<25 \%$ & $35(57.4)$ & $11(84.6)$ & $24(50.0)$ & 0.030 & $21(58.3)$ & $3(25.0)$ & 0.093 \\
\hline $25-50 \%$ & $22(36.1)$ & $2(15.4)$ & $20(41.7)$ & 0.109 & $14(38.9)$ & $6(50.0)$ & 0.365 \\
\hline$>50 \%$ & $4(6.6)$ & $0(0)$ & $4(8.3)$ & 0.569 & $1(2.8)$ & $3(25.0)$ & 0.043 \\
\hline \multicolumn{8}{|l|}{ Other findings } \\
\hline Pleural effusion & $5(8.2)$ & $0(0)$ & $5(10.4)$ & 0.575 & $2(5.6)$ & $3(25.0)$ & 0.092 \\
\hline $\begin{array}{l}\text { Mediastinal lymph node } \\
\text { enlargement }\end{array}$ & $2(3.3)$ & $0(0)$ & $2(4.2)$ & 1.000 & $2(5.6)$ & $0(0)$ & 1.000 \\
\hline
\end{tabular}

Data are number (\%) of patients. Group 1: discharged patients; group 2: patients hospitalized in general wards; group 3: patients admitted to ICU $G G O$ ground glass opacities

*All hospitalized patients; includes groups 2 and 3

${ }^{a} P$ value refers to group 1 vs all hospitalized patients (groups 2 and 3)

${ }^{\mathrm{b}} P$ value refers to group 2 vs group 3

Italicized numbers represent statistically significant $P$ values

interlobular septal thickening, which may occur due to interstitial pulmonary edema or interstitial injury, was observed in $39.3 \%$ of the cases. As previously reported [18], interlobular septal thickening is associated with more severe disease and, in our study, it occurred more frequently in ICU admitted patients and also correlated with longer stay in the ICU.

The same CT features (interlobular septal thickening and more extensive lung involvement) also correlated with lower $\mathrm{SpO}_{2}$ and elevated CRP, probably due to the same reasons. Diffuse distribution (rather than focal or multifocal), consolidation, and pleural effusion were associated with more severe disease in previous reports $[15,17,31]$. In our study, these findings correlated with clinical and laboratorial features of severe disease but did not correlate with patients' outcome. Despite being considered an uncommon finding in COVID-19, pleural effusion occurred between none and more than $20 \%$ of the cases in previous studies $[15,17,31,32]$. In our study, mild pleural effusion was seen in $8.2 \%$ of the cases. Although not clearly understood, pleural effusion could be related not only to the virulence of the disease but also to comorbidities, such as heart or renal failure, malignancies, and pregnancy [33]. 


\section{Study limitations}

This is a single-center study, with limited sample size. Our sample included only symptomatic patients who underwent the emergency department, and, despite the low sensitivity of RT-PCR, patients with negative test were excluded from the study, even those cases with highly suspicious CT findings.

Clinical data and laboratorial tests depended on the physician on duty's decision in this retrospective study. Several other laboratorial tests associated with severe illness could have been analyzed and correlated with CT findings if available. Also, comorbidities were not included in our analysis, and co-infections could not be completely excluded.

CT scans were performed in different times after the onset of symptoms. Early-performed CTs frequently show only few changes, which do not necessarily mean a benign prognosis. A study with patients within the same phase of the disease could be more accurate in the recognition of potentially severe cases. Moreover, none of the patients of our study died; a larger cohort of patients might enable a study correlating imaging findings with mortality.

\section{Conclusion}

Extension of lung disease estimated by visual analysis of CT showed correlation with patients' outcome and also with clinical and laboratorial signs related to severe COVID-19 disease. Interlobular septal thickening was a CT feature more frequently seen on ICU-admitted patients than in ward patients and also correlated with longer hospitalization time on ICU. Diffuse distribution, interlobular septal thickening, consolidation, and pleural effusion correlated with clinical and laboratorial features associated with severe illness.

Data availability All data generated or analyzed during this study are included in this published article.

Code availability Not applicable.

\section{Compliance with ethical standards}

Conflict of interest The authors declare that they have no conflict of interest.

Ethics approval The Ethics Committee of Sírio Libanês Hospital approved the study protocol.

Consent to participate The informed consent was waived by the ethics committee for this retrospective study 


\section{References}

1. Organization WH. Coronavirus disease (COVID-19) outbreak situation [Web Site]. 2020 [Retrived from https://www.who.int/ emergencies/diseases/novel-coronavirus-2019]. Accessed 31 Aug 2020

2. Su S, Wong G, Shi W, Liu J, Lai ACK, Zhou J, Liu W, Bi Y, Gao GF (2016) Epidemiology, genetic recombination, and pathogenesis of coronaviruses. Trends Microbiol 24(6):490-502

3. Li Q, Guan X, Wu P, Wang X, Zhou L, Tong Y, Ren R, Leung KSM, Lau EHY, Wong JY, Xing X, Xiang N, Wu Y, Li C, Chen Q, Li D, Liu T, Zhao J, Liu M, Tu W, Chen C, Jin L, Yang R, Wang Q, Zhou S, Wang R, Liu H, Luo Y, Liu Y, Shao G, Li H, Tao Z, Yang Y, Deng Z, Liu B, Ma Z, Zhang Y, Shi G, Lam TTY, Wu JT, Gao GF, Cowling BJ, Yang B, Leung GM, Feng Z (2020) Early transmission dynamics in Wuhan, China, of novel coronavirus-infected pneumonia. N Engl J Med 382(13):1199-1207

4. Yi Y, Lagniton PNP, Ye S, Li E, Xu RH (2020) COVID-19: what has been learned and to be learned about the novel coronavirus disease. Int J Biol Sci 16(10):1753-1766

5. Lomoro P, Verde F, Zerboni F, Simonetti I, Borghi C, Fachinetti C, Natalizi A, Martegani A (2020) COVID-19 pneumonia manifestations at the admission on chest ultrasound, radiographs, and CT: single-center study and comprehensive radiologic literature review. Eur J Radiol Open 7:100231

6. Watson J, Whiting PF, Brush JE (2020) Interpreting a covid-19 test result. BMJ. 369:m1808

7. Fang Y, Zhang H, Xie J, Lin M, Ying L, Pang P, et al. (2020) Sensitivity of chest CT for COVID-19: comparison to RT-PCR. Radiology ;200432

8. Guan WJ, Ni ZY, Hu Y, Liang WH, Ou CQ, He JX, Liu L, Shan H, Lei CL, Hui DSC, du B, Li LJ, Zeng G, Yuen KY, Chen RC, Tang CL, Wang T, Chen PY, Xiang J, Li SY, Wang JL, Liang ZJ, Peng YX, Wei L, Liu Y, Hu YH, Peng P, Wang JM, Liu JY, Chen Z, Li G, Zheng ZJ, Qiu SQ, Luo J, Ye CJ, Zhu SY, Zhong NS (2020) Clinical characteristics of coronavirus disease 2019 in China. N Engl J Med 382(18):1708-1720

9. Huang C, Wang Y, Li X, Ren L, Zhao J, Hu Y, Zhang L, Fan G, Xu J, Gu X, Cheng Z, Yu T, Xia J, Wei Y, Wu W, Xie X, Yin W, Li H, Liu M, Xiao Y, Gao H, Guo L, Xie J, Wang G, Jiang R, Gao Z, Jin Q, Wang J, Cao B (2020) Clinical features of patients infected with 2019 novel coronavirus in Wuhan, China. Lancet 395(10223):497506

10. Wu C, Chen X, Cai Y, Xia J, Zhou X, Xu S, et al. (2020) Risk factors associated with acute respiratory distress syndrome and death in patients with coronavirus disease 2019 pneumonia in Wuhan, China. JAMA Intern Med

11. Akl EA, Blazic I, Yaacoub S, Frija G, Chou R, Appiah JA, et al. (2020) Use of chest imaging in the diagnosis and management of COVID-19: a WHO rapid advice guide. Radiology 203173

12. Majidi H, Bani-Mostafavi ES, Mardanshahi Z, Godazandeh F, Ghasemian R, Heydari K, Alizadeh-Navaei R (2020) Highresolution computed tomography finding in 552 patients with symptomatic COVID-19: first report from north of Iran. Emerg Radiol

13. Tabatabaei SMH, Talari H, Gholamrezanezhad A, Farhood B, Rahimi H, Razzaghi R, Mehri N, Rajebi H (2020) A low-dose chest CT protocol for the diagnosis of COVID-19 pneumonia: a prospective study. Emerg Radiol

14. Simpson S, Kay FU, Abbara S, Bhalla S, Chung JH, Chung M, et al. (2020) Radiological Society of North America Expert
Consensus statement on reporting chest CT findings related to COVID-19. Endorsed by the Society of Thoracic Radiology, the American College of Radiology, and RSNA. J Thorac Imaging

15. Zhao W, Zhong Z, Xie X, Yu Q, Liu J. (2020) Relation between chest $\mathrm{CT}$ findings and clinical conditions of coronavirus disease (COVID-19) pneumonia: a multicenter study. AJR Am J Roentgenol 1-6

16. Salehi S, Abedi A, Balakrishnan S, Gholamrezanezhad A, Coronavirus Disease (2019) (COVID-19): a systematic review of imaging findings in 919 patients. AJR Am J Roentgenol 2020:1-7

17. Yu M, Xu D, Lan L, Tu M, Liao R, Cai S, Cao Y, Xu L, Liao M, Zhang X, Xiao SY, Li Y, Xu H (2020) Thin-section chest CT imaging of coronavirus disease 2019 pneumonia: comparison between patients with mild and severe disease. Radiology: Cardiothoracic Imaging 2:1-21

18. Yang X, Yu Y, Xu J, Shu H, Xia J, Liu H et al (2020) Clinical course and outcomes of critically ill patients with SARS-CoV-2 pneumonia in Wuhan, China: a single-centered, retrospective, observational study. Lancet Respir Med 8(5):475-481

19. Wang D, Hu B, Hu C, Zhu F, Liu X, Zhang J, et al. (2020) Clinical Characteristics of 138 Hospitalized Patients With 2019 Novel coronavirus-infected pneumonia in Wuhan, China JAMA

20. Wu Z, McGoogan JM (2020) Characteristics of and important lessons from the coronavirus disease 2019 (COVID-19) outbreak in China: summary of a report of 72314 cases from the Chinese Center for Disease Control and Prevention. JAMA. 323:1239-1242

21. Grasselli G, Pesenti A, Cecconi M. (2020) Critical care utilization for the COVID-19 outbreak in Lombardy, Italy: early experience and forecast during an emergency response. JAMA

22. Richardson S, Hirsch JS, Narasimhan M, Crawford JM, McGinn T, Davidson KW, and the Northwell COVID-19 Research Consortium, Barnaby DP, Becker LB, Chelico JD, Cohen SL, Cookingham J, Coppa K, Diefenbach MA, Dominello AJ, DuerHefele J, Falzon L, Gitlin J, Hajizadeh N, Harvin TG, Hirschwerk DA, Kim EJ, Kozel ZM, Marrast LM, Mogavero JN, Osorio GA, Qiu M, Zanos TP (2020) Presenting characteristics, comorbidities, and outcomes among 5700 patients hospitalized with COVID-19 in the New York City area. JAMA. 323:2052-2059

23. Livingston E, Bucher K (2020) Coronavirus disease 2019 (COVID19) in Italy. JAMA. 323:1335

24. Tan C, Huang Y, Shi F, Tan K, Ma Q, Chen Y, Jiang X, Li X (2020) C-reactive protein correlates with computed tomographic findings and predicts severe COVID-19 early. J Med Virol 92: 856-862

25. Wang L (2020) C-reactive protein levels in the early stage of COVID-19. Med Mal Infect 50:332-334

26. Gong J, Ou J, Qiu X, Jie Y, Chen Y, Yuan L, et al. (2020) A tool to early predict severe Corona Virus Disease 2019 (COVID-19) : a multicenter study using the risk nomogram in Wuhan and Guangdong, China. Clin Infect Dis

27. Frater JL, Zini G, d'Onofrio G, Rogers HJ (2020) COVID-19 and the clinical hematology laboratory. Int J Lab Hematol 42:11-18

28. Lippi G, Plebani M (2020) Laboratory abnormalities in patients with COVID-2019 infection. Clin Chem Lab Med 58:1131-1134

29. Wang K, Kang S, Tian R, Zhang X, Wang Y (2020) Imaging manifestations and diagnostic value of chest $\mathrm{CT}$ of coronavirus disease 2019 (COVID-19) in the Xiaogan area. Clin Radiol 75(5): 341-347

30. Lyu P, Liu X, Zhang R, Shi L, Gao J. (2020) The performance of chest CT in evaluating the clinical severity of COVID-19 pneumonia: identifying critical cases based on CT characteristics. Investig Radiol 
31. Tabatabaei SMH, Talari H, Moghaddas F, Rajebi H (2020) Computed tomographic features and short-term prognosis of coronavirus disease 2019 (COVID-19) pneumonia: a single-center study from Kashan, Iran. Radiol Cardiothorac Imaging 2(2): e200130

32. Ng M-Y, Lee EYP, Yang J, Yang F, Li X, Wang H, Lui MMS, Lo CSY, Leung B, Khong PL, Hui CKM, Yuen KY, Kuo MD (2020) Imaging profile of the COVID-19 infection: radiologic findings and literature review. Radiol Cardiothorac Imaging 2(1):e200034
33. Gong X, Song L, Li H, Li L, Jin W, Yu K, Zhang X, Li H, Ke HN, Lu ZY (2020) CT characteristics and diagnostic value of COVID19 in pregnancy. PLoS One 15(7):e0235134

Publisher's note Springer Nature remains neutral with regard to jurisdictional claims in published maps and institutional affiliations. 